\title{
Magnetic Circuit Analyses and Turning Chatter Suppression Based on a Squeeze-Mode Magnetorheological Damping Turning Tool
}

\author{
Yongliang Zhang, ${ }^{1,2}$ Norman M. Wereley, ${ }^{2}$ Wei Hu, ${ }^{2}$ Ming Hong, ${ }^{1}$ and Wei Zhang ${ }^{1}$ \\ ${ }^{1}$ Department of Mechanical Engineering, University of Shanghai for Science and Technology, Shanghai 200093, China \\ ${ }^{2}$ Department of Aerospace Engineering, University of Maryland, College Park, MD 20742, USA \\ Correspondence should be addressed to Yongliang Zhang; yongliangz7377@163.com
}

Received 25 June 2014; Accepted 5 September 2014

Academic Editor: Weihua Li

Copyright (C) 2015 Yongliang Zhang et al. This is an open access article distributed under the Creative Commons Attribution License, which permits unrestricted use, distribution, and reproduction in any medium, provided the original work is properly cited.

\begin{abstract}
As a smart material, magnetorheological fluid (MRF) has been utilized in fields including civil engineering and automotive engineering, and so on. In this study, the MR damping turning tool based on the squeeze-mode was developed to improve the vibration resistance of the tool system on the lathe. The 3D magnetic circuit simulations of the damper were performed. The influences of damper structural parameters, such as coil positions, plate thicknesses, and others, on the magnetic induction strength were investigated. Orthogonal experiments were carried out and the optimal combination of damper parameters was determined. The chatter suppressive experiments were carried out to evaluate the performance of the MR damping turning tool.
\end{abstract}

\section{Introduction}

Turning is a primary machining method in manufacturing industries. However, turning vibration is a significant factor limiting machining efficiency and preventing improvements in product quality. To reduce turning vibration, various dampers have been proposed in succession, including damping absorbers, dynamic vibration absorbers, friction dampers, and impact dampers [1]. In recent years, with the development of intelligent materials, the corresponding dampers have been developed by using unique properties of these kinds of material and researchers have paid more attention to this field.

As a semiactive control damper, magnetorheological (MR) dampers provide both adaptive and fail-safe passive force. In addition, they also possess advantages such as simplicity of structure, continuous adjustability of damping force, quick response, large output force, and low power consumption [2]. MR dampers have been utilized in fields including civil engineering, machine control, and automotive engineering [3-7]. It was reported that a magnetorheological semiactive suspension system installed on a heavy
Humvee increased cross-country speed by $30 \%$ to $40 \%$. Jiang and Christenson proposed a large-scale MR damper for highway bridges, and the simulation and experimental results indicated that the peak and dynamic responses of a bridge were effectively reduced and the fatigue life of the structure was extended. Mei et al. developed an innovative magnetorheological (MR) fluid-controlled boring bar for chatter suppression.

The magnetic circuit design of a magnetorheological damper is critical to improve the working performance of the damper. A rational design of the circuit provides sufficient magnetic flux density for the damper in its operational environment, subsequently generating the required damping force [8-12]. Meanwhile, it is also necessary to avoid magnetic saturation. Nguyen and Choi generated braking force with a hybrid concept of magnetic circuit (using both axial and radial magnetic flux). Aiming at a flow-mode magnetorheological damper, by combining the power consumption of the coil and the piston volume as objective functions, Zheng et al. studied the optimal structural design of magnetorheological dampers which minimised the objective functions. Nguyen and Choi proposed a new approach to analyze the magnetic 


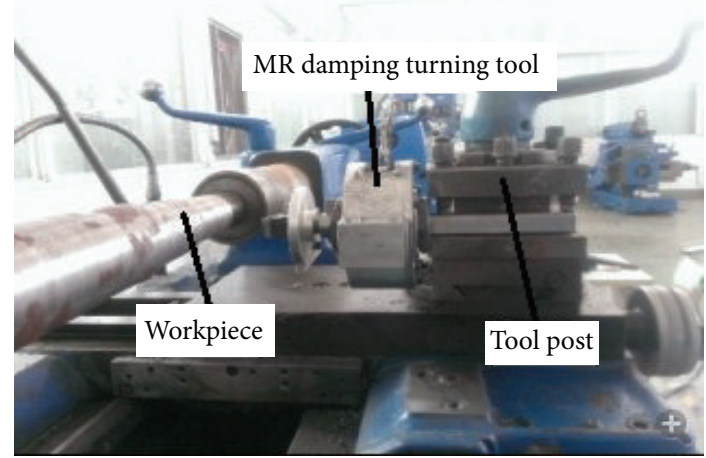

FIgURE 1: The photograph of MR damping turning tool.

circuit of an MR brake and used the approach to explore an engineering optimization problem.

Currently, much research on MR dampers has been carried out based on shearing and flow modes $[13,14]$. The contribution of this work lies in two aspects. One is that a squeeze-mode magnetorheological damping turning tool was developed to effectively reduce vibrations in cylindrical turning since the yield strength of magnetorheological materials generated in squeeze mode is larger than that in shear mode, and the volume of a squeezed-mode MRF damper is smaller than that in other working modes. The other contribution is that the three-dimensional (3D) finite element simulations of the magnetic circuit of the damper were conducted, and the orthogonal experimental analyses were used to investigate the magnetic induction strength. With this method, the influence of different structural parameters on the magnetic flux density in the pole gap of the damper can be evaluated, which is helpful for the determination of the structural parameters MRF dampers. In this study, the key parameters influencing the damper were determined subsequently. Finally, cylindrical turning damping experiments were carried out using the MR damping turning tool with above key parameters to verify its effectiveness.

\section{The Structure and Working Principle of the Magnetorheological Damping Turning Tool}

In the cylindrical turning process of axis parts, major vibration exists in the normal direction of the turning surface of the parts, namely, the $y$-direction in Figure 2. In this study, a magnetorheological damper was installed on the rod of a turning tool to improve the vibration resistance of the tool system. The installation photograph of the magnetorheological damping turning tool is shown in Figure 1.

Since the yield stress of a magnetorheological fluid in squeeze mode is higher than that in the shear mode in a magnetic field, the squeeze mode was used as the working mode of this magnetorheological turning tool. The structure of the magnetorheological damper is depicted in Figure 2. In the damper, the rod axle (1) replaced the original rod of the cylindrical turning tool and was rigidly connected to the cylindrical turning tool head using a Morse taper,

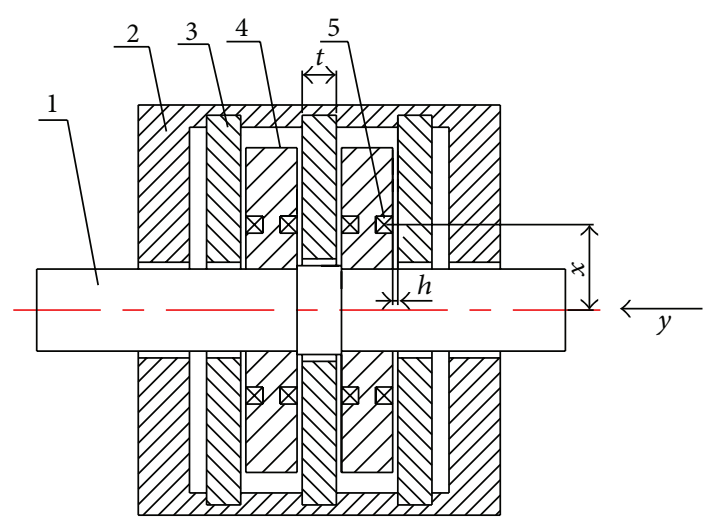

FIGURE 2: Structural diagram of MR damping turning tool.

the iron core (4) was interference fitted with the rod axle (1) and synchronously vibrated with the turning tool during the turning process, and the pole plate (3) was rigidly connected to the shell (2).

After applying current on the coil (5), magnetic field was generated in the damper. The direction of the magnetic flux lines between the two poles, namely, iron core (4) and pole plate (3), parallels the $y$-direction in Figure 2. Under action of the magnetic field, the magnetorheological fluid was polarised to chains along the $y$-direction. When vibration occurred during cylindrical turning, the iron core (4) and the turning tool underwent reciprocal motion along the $y$ direction relative to pole plate (3), and subsequently this motion squeezed the polarized chains of magnetorheological fluid. With the change of the applied current the squeeze yield strength of the magnetorheological fluid varied such that the damping and stiffness generated by MR damping turning tool could be controlled, thus restraining the vibration of the iron core (4) and the turning tool.

\section{Simulation Analyses of the Magnetic Circuit under Different Parameters}

The magnetic induction in the pole gap of the magnetorheological damper was influenced by varied parameters, such as coil position, rod axis material, pole plate thickness, and pole gap. In order to investigate the effect of these parameters on magnetic induction, the 3D simulations of magnetic induction were performed under different parameters using Ansoft Maxwell software.

3.1. Preprocessing. A 3D model of the damper was established using Solidworks software. Figure 3(a) shows the meshing results of the outside surface, and Figure $3(\mathrm{~b})$ shows the meshing results of the axial section shown in Figure 2 after $3 \mathrm{D}$ meshing. In simulation, the type of current was defined as stranded, and the total current was inputted by $N I=120 \times$ $2 \mathrm{~A}=240 \mathrm{~A}$.

3.2. The Influence of Coil Position. The simulations of magnetic induction strength under different coil positions were 


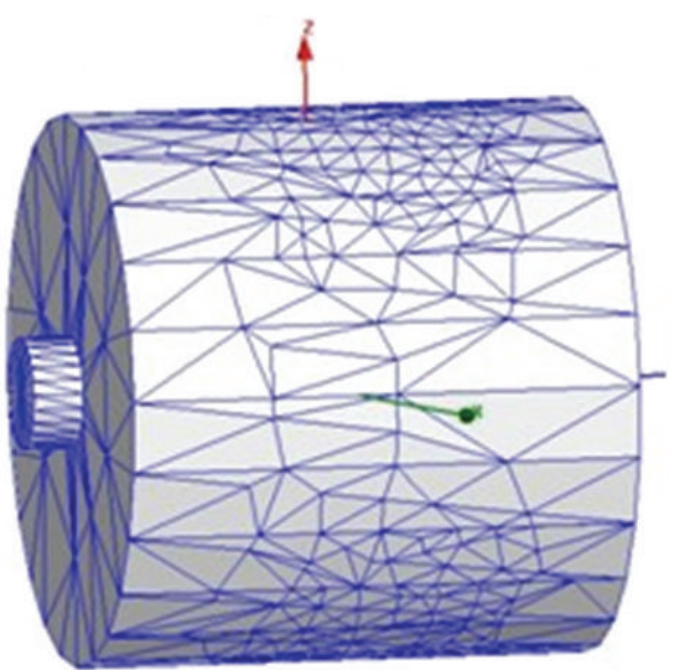

(a) Meshing result on surface of model

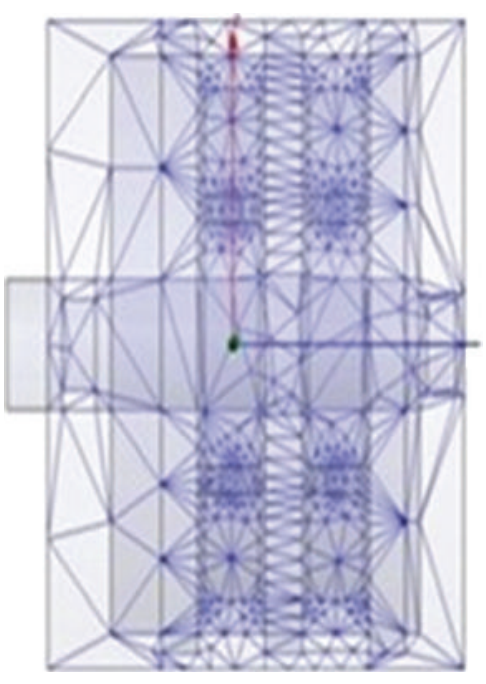

(b) Meshing result on the selected section

FIGURE 3: Chart of 3D meshing results.

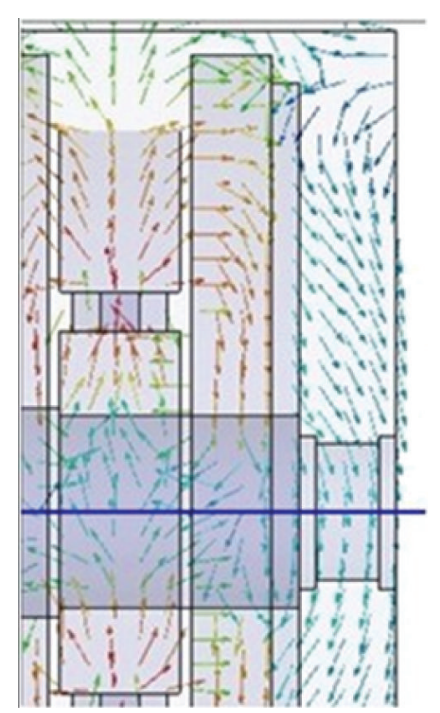

(a) Aluminum

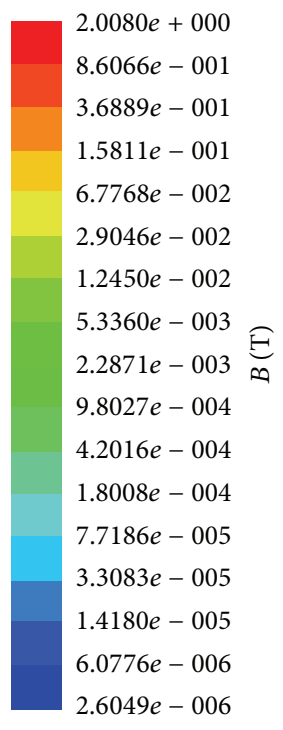

$2.6049 e-006$

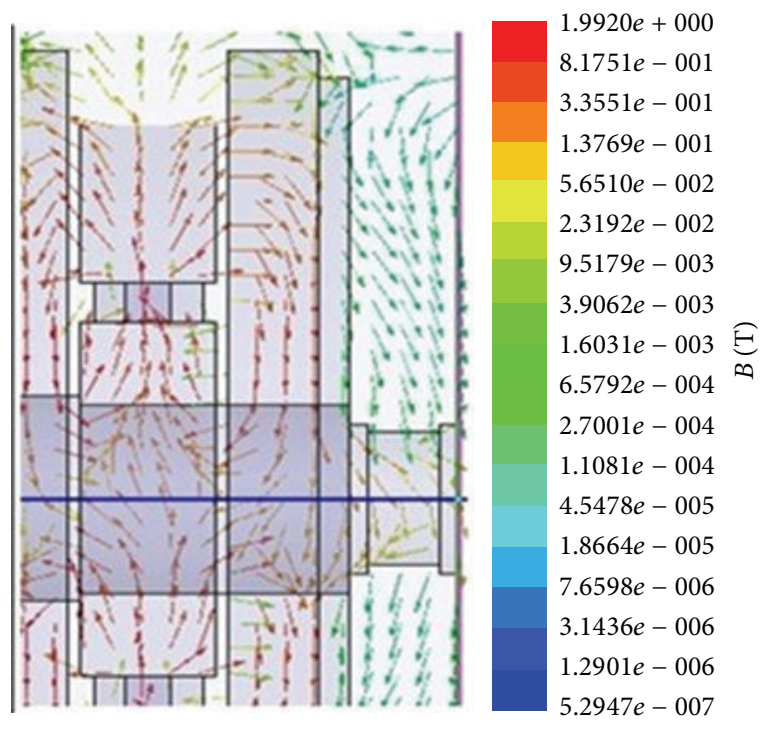

(b) $8^{\#}$ steel

FIgURE 4: Cloud charts of magnetic lines of force for arbor shaft in aluminum and $8^{\#}$ steel.

carried out, and their maximum values of magnetic induction strength between the gaps are listed in Table 1 . The $x$ indicates the radial distance from the axis of rod axle to the coil, shown in Figure 2. The results display that the magnetic induction strength increases as the distance between the coil and the axis increases.

3.3. The Influence of Rod Axle Material. The permeability of material has strong effect on the magnetic induction strength. The magnetic induction strengths with different material of rod axle are simulated and their maximum values at gap are listed in Table 2, in which the permeability of varied materials
TABLE 1: Simulated results of magnetic induction strength at coil's different positions.

\begin{tabular}{lcc}
\hline Number & $\begin{array}{c}\text { Position of coil } \\
x / \mathrm{mm}\end{array}$ & $\begin{array}{c}\text { Magnetic induction strength } \\
B_{\max } / T\end{array}$ \\
\hline 1 & 20 & 0.258 \\
2 & 22.5 & 0.369 \\
3 & 25 & 0.476 \\
\hline
\end{tabular}

tends to increase in order of aluminium, $45^{\#}$, and $8^{\#}$. The results show that the lower the permeability of rod axle's material, the higher the magnetic induction strength. 
TABLE 2: Simulated results of magnetic induction strength for different material of rod axle.

\begin{tabular}{lcc}
\hline Number & Material of rod axle & $\begin{array}{c}\text { Magnetic induction strength } \\
B_{\max } / T\end{array}$ \\
\hline 1 & Aluminium & 0.369 \\
2 & $45^{\#}$ & 0.354 \\
3 & $8^{\#}$ & 0.336 \\
\hline
\end{tabular}

TABLE 3: Simulated results of magnetic induction strength for different plate thickness.

\begin{tabular}{lcc}
\hline Number & $\begin{array}{c}\text { Plate thickness } \\
t / \mathrm{mm}\end{array}$ & $\begin{array}{c}\text { Magnetic induction strength } \\
B_{\max } / T\end{array}$ \\
\hline 1 & 8 & 0.252 \\
2 & 10 & 0.369 \\
3 & 12 & 0.269 \\
\hline
\end{tabular}

TABLE 4: Simulated results of magnetic induction strength for different width of gap.

\begin{tabular}{lcc}
\hline Number & $\begin{array}{c}\text { Width of gap } \\
h / \mathrm{mm}\end{array}$ & $\begin{array}{c}\text { Magnetic induction strength } \\
B_{\max } / T\end{array}$ \\
\hline 1 & 1.5 & 0.369 \\
2 & 1.8 & 0.278 \\
3 & 2.0 & 0.246 \\
\hline
\end{tabular}

The cloud charts of magnetic lines of force with aluminium and $8^{\#}$ are, respectively, given in Figures 4(a) and 4(b). In Figure 4(a), the strength of magnetic lines of force is weak through the rod axle and only reaches the $10^{-4}$ orders of magnitude. On the other hand, when the material of the rod axle is $8^{\#}$, the strength of magnetic lines of force through the rod axle increases about 100 times.

3.4. The Influence of Plate Thickness. The simulations of magnetic induction strength with different value of plate thickness were performed, and their maximum values are listed in Table 3. The plate thickness $t$ is given in Figure 2. In Table 3, the relationship between plate thickness and magnetic induction strength is not monotonic. For the damper in this paper, the magnetic induction strength is maximized when the plate thickness is $10 \mathrm{~mm}$, and the magnetic induction strength decreases when the plate thickness either increases or decreases.

3.5. The Influence of the Width of Pole Gap. The magnetic induction strengths with different width of gap were simulated, and the results are listed in Table 4, in which the thickness of plate is $10 \mathrm{~mm}$. The results demonstrate that the narrower the gap is, the larger the magnetic induction strength is. The width of gap $h$ is displayed in Figure 2.

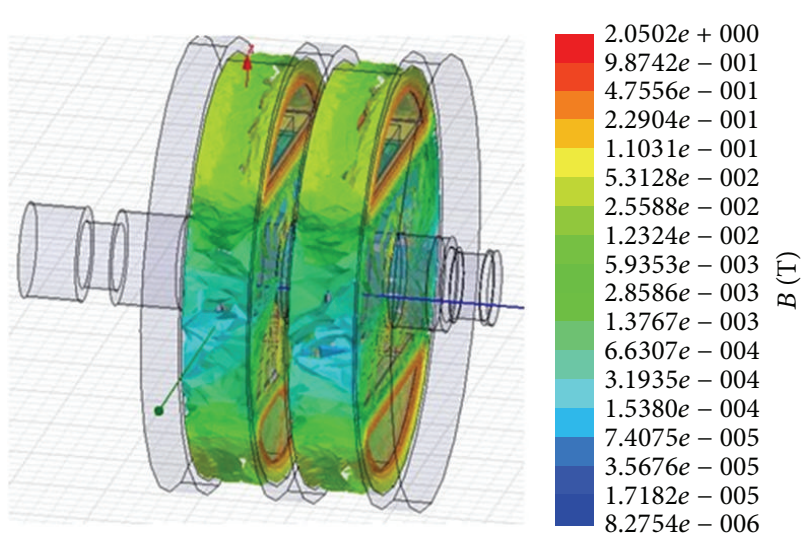

Figure 5: 3D simulated result of magnetic induction strength.

\section{Magnetic Circuit Analyses Using Multifactor Orthogonal Experiment}

To determine the optimal structural size of the damper, an orthogonal experiment was carried out using orthogonal table of four factors and three levels $\mathrm{L}_{9}\left(3^{4}\right)$. With this experiment, the structural parameter of damper with the strongest or weakest effect on the magnetic induction $B$ can be analysed, and the optimal combination of different parameters can be achieved. Table 5 shows the factors and levels in the experiment, and Table 6 lists the experimental scheme and the simulating results under corresponding factors and levels.

In Table 6, the variances are used to evaluate the impact of each factor on magnetic induction strength. Generally, the larger variance demonstrates that the corresponding factor has a stronger influence on the experimental results. The data in Table 6 indicate that the material of rod axle is the main factor influencing the magnetic induction strength, and the factors influencing the magnetic induction in the descending order were the material of rod axle, pole gap, coil position, and pole plate thickness. The optimal combination to acquire the appropriate magnetic induction was as follows: the rod axle's material is aluminium and the distance from coil to the axial symmetry centre of the damper was $x=25 \mathrm{~mm}$ at pole plate thickness of $10 \mathrm{~mm}$ and pole gap of $1.5 \mathrm{~mm}$.

Figures 5 and 6 show the 3D simulated distribution of the magnetic induction of the damper and the distribution of the magnetic induction on the section displayed in Figure 2 according to the optimal structural parameters stated above, respectively. It can be observed that the maximum magnetic induction in the pole gap reached $0.54 \mathrm{~T}$, which complied with design requirements.

\section{Damping Experiments Based on the Magnetorheological Damping Turning Tool}

To verify the chatter suppressive effect of the magnetorheological damping turning tool, it was manufactured and installed on a CA6140 lathe carriage, and the chatter suppressive experiments were carried out. The experimental 
TABLE 5: The factors and levels.

\begin{tabular}{lcccc}
\hline Level & & \multicolumn{2}{c}{ Factor } & Rod axle material \\
\hline 1 & Coil position $x / \mathrm{mm}$ & Plate thickness $t / \mathrm{mm}$ & 1.5 & Aluminum \\
2 & 20 & 8 & 1.8 & $45^{\#}$ \\
3 & 22.5 & 10 & 2.0 & $8^{\#}$ \\
\hline
\end{tabular}

TABLE 6: The experimental scheme and results.

\begin{tabular}{|c|c|c|c|c|c|}
\hline Factor & $x$ & $t$ & $h$ & Material & \multirow[t]{2}{*}{ Magnetic induction $B / T$} \\
\hline Test number & \multicolumn{4}{|c|}{ Level number } & \\
\hline 1 & 1 & 1 & 1 & 1 & 0.35728 \\
\hline 2 & 1 & 2 & 2 & 2 & 0.31623 \\
\hline 3 & 1 & 3 & 3 & 3 & 0.29431 \\
\hline 4 & 2 & 1 & 2 & 3 & 0.28730 \\
\hline 5 & 2 & 2 & 3 & 1 & 0.34744 \\
\hline 6 & 2 & 3 & 1 & 2 & 0.32650 \\
\hline 7 & 3 & 1 & 3 & 2 & 0.33056 \\
\hline 8 & 3 & 2 & 1 & 3 & 0.35822 \\
\hline 9 & 3 & 3 & 2 & 1 & 0.34830 \\
\hline$k_{1}$ & 0.32261 & 0.32505 & 0.34733 & 0.35101 & \\
\hline$k_{2}$ & 0.32041 & 0.34063 & 0.31728 & 0.32443 & \\
\hline$k_{3}$ & 0.34569 & 0.32304 & 0.32410 & 0.31477 & \\
\hline Variance & 0.02528 & 0.01759 & 0.03005 & 0.03624 & \\
\hline Descending order & \multicolumn{5}{|c|}{ Material, $h, x, t$} \\
\hline Optimal scheme & \multicolumn{5}{|c|}{ material $_{1} h_{1} x_{3} t_{2}$} \\
\hline
\end{tabular}

Note: $k_{i}=\operatorname{sum}\left(B_{i}\right) / s$, where $B_{i}$ is magnetic induction when the level number is $i$ and $s$ is the number of level $i$ in that column, $i=1,2,3$.

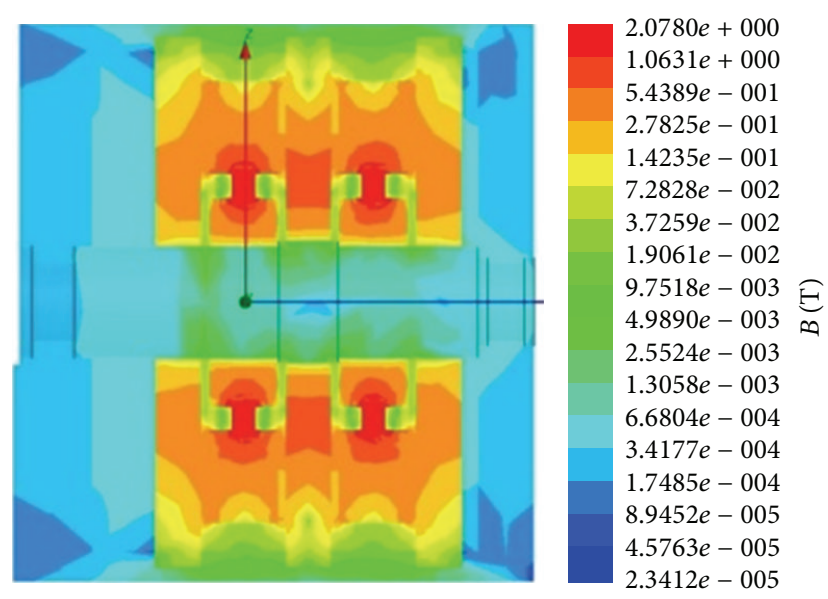

FIGURE 6: Results of magnetic induction strength on the selected section.

system block diagram is shown in Figure 7. The MRF-132DG developed by the American LORD company was used in the experiments, and the workpiece was $45^{\#}$ steel bar stock $(600 \mathrm{~mm}$ in length and $60 \mathrm{~mm}$ in diameter). The turning parameters were spindle speed $n=320 \mathrm{rpm}$, feed rate $f=$ $0.08 \mathrm{~mm} / \mathrm{r}$, and depth of turning $a_{p}=0.4 \mathrm{~mm}$.

Figures 8(a) and 8(b) show the time-domain signals of the acceleration of the magnetorheological damping turning tool in the $y$-direction without current and with $2 \mathrm{~A}$ applied current on each coil, respectively. Figure 8(a) shows that the vibration amplitude of the acceleration signal increased at time $t=4 \mathrm{~s}$ and the amplitude increased with the continuation of the process. Figure 8(b) shows the results of imposing $2 \mathrm{~A}$ on the damper at $t=6 \mathrm{~s}$. The amplitude of the acceleration signal decreased at $t=20 \mathrm{~s}$ after imposing current and a substantially reduced amplitude was maintained thereafter.

In the frequency domain, Figure 9(a) indicates that the main vibration frequency of the vibration signal before imposing current was approximately $255 \mathrm{~Hz}$ and the peak of power spectrum density was approximately $1.1 \times 10^{7} \mathrm{~V}$. Figure 9(b) shows the dominant frequency of the vibration signal after imposing current, increased to approximately $466 \mathrm{~Hz}$. This indicated that the stiffness of the system was enhanced and the peak of the dominant mode of the system had been reduced to $7.1 \times 10^{4} \mathrm{~V}$, which was $7 \%$ of the peak value when the current was not applied.

These time- and frequency-domain analyses showed that the developed magnetorheological damping turning tool exhibited a significant restraining effect on turning vibration.

\section{Conclusions}

To reduce the vibration in cylindrical turning, a magnetorheological damping turning tool based on the squeeze mode was designed and manufactured. On the basis of 


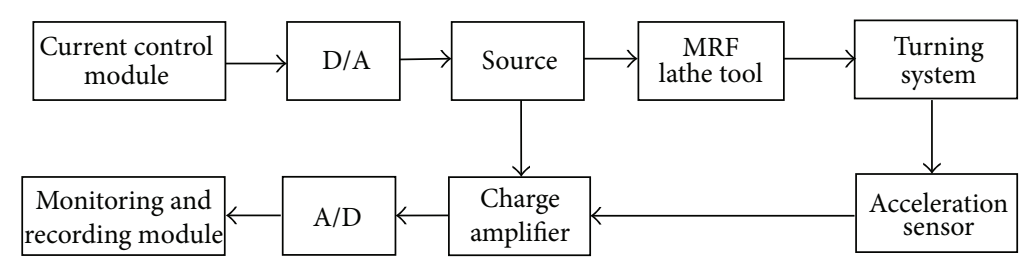

FIGURE 7: The system block diagram of turning damping experiment.

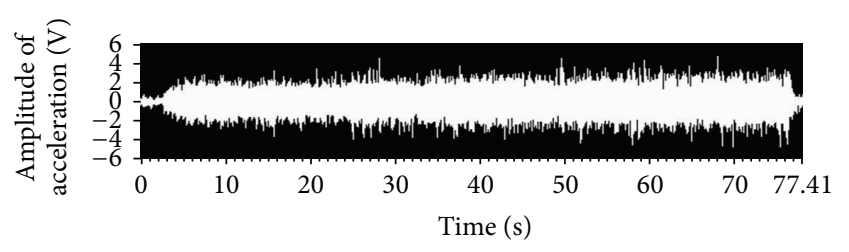

(a)

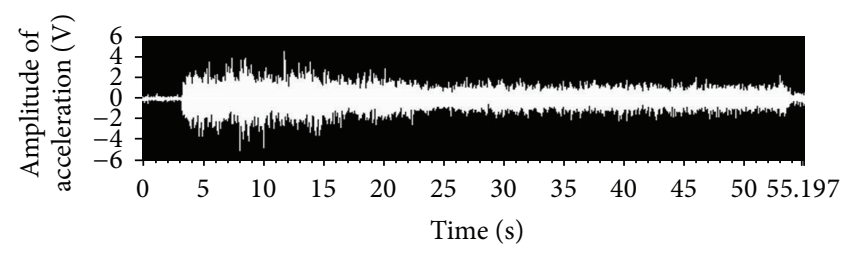

(b)

FIgURE 8: The time-domain signals of acceleration without current and 2 A current on each coil.

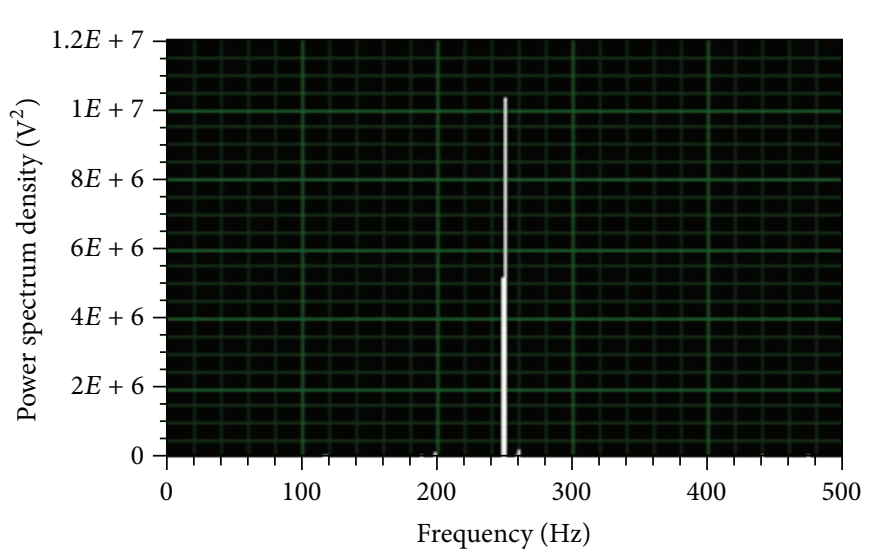

(a)

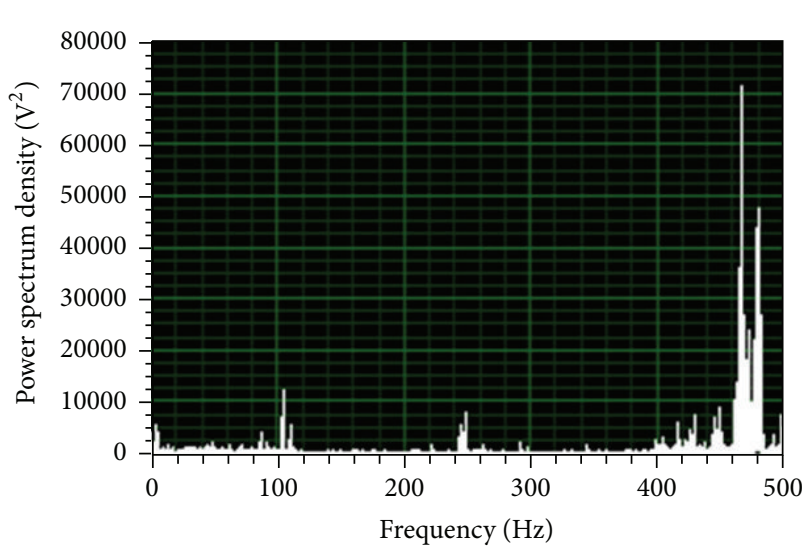

(b)

FIGURE 9: The power spectrum density of corresponding acceleration signals before and after imposing current.

its structural design, 3D finite element simulations of the magnetic induction of the damper were carried out with different parameters according to the basic principles of electromagnetism. It shows that the magnetic field strength in the gap increases as the distance between the axis of the rod axle and coil increases, the permeability of the rob axle decreases and the gap thickness decreases. As to the magnetic pole thickness, the relationship between it and magnetic induction strength is not monotonous.

Furthermore, these simulation data were utilized in the orthogonal experiment. The results indicated that the factors influencing the magnetic induction of this magnetorheological damping turning tool, in decreasing order, were pole gap, coil position, and plate thickness. Therefore, the optimal structural parameters of the damping turning tool were determined.

Afterwards, chatter suppression experiments were undertaken during cylindrical turning using the prototyped magnetorheological damping turning tool. The experimental data from both time- and frequency-domains verified that the magnetorheological damping turning tool, based on the squeeze mode, can significantly reduce turning vibration.

\section{Conflict of Interests}

The authors declare that there are no conflicts of interests regarding the publication of this paper.

\section{Acknowledgment}

The project "Magnetorheological fluid mechanics modeling and its coupled damping mechanism of turning system with variable parameters" was supported by the National Natural Science Foundation of China (no. 51205255).

\section{References}

[1] M. Siddhpura and R. Paurobally, "A review of chatter vibration research in turning," International Journal of Machine Tools and Manufacture, vol. 61, pp. 27-47, 2012. 
[2] G. Park, M. T. Bement, D. A. Hartman, R. E. Smith, and C. R. Farrar, "The use of active materials for machining processes: a review," International Journal of Machine Tools \& Manufacture, vol. 47, no. 15, pp. 2189-2206, 2007.

[3] S. H. Ha, M.-S. Seong, and S.-B. Choi, "Design and vibration control of military vehicle suspension system using magnetorheological damper and disc spring," Smart Materials and Structures, vol. 22, no. 6, Article ID 065006, 2013.

[4] A. Farjoud, R. Taylor, E. Schumann, and T. Schlangen, "Advanced semi-active engine and transmission mounts: tools for modelling, analysis, design, and tuning," Vehicle System Dynamics, vol. 52, no. 2, pp. 218-243, 2014.

[5] Z. Jiang and R. Christenson, "Experimental verification of an MR damper controlled highway bridge," in Proceedings of the 19th Analysis and Computation Specialty Conference, pp. 347358, Orlando, Fla, USA, May 2010.

[6] D. Sathianarayanan, L. Karunamoorthy, J. Srinivasan, G. S. Kandasami, and K. Palanikumar, "Chatter suppression in boring operation using magnetorheological fluid damper," Materials and Manufacturing Processes, vol. 23, no. 4, pp. 329-335, 2008.

[7] D. Mei, Z. Yao, T. Kong, and Z. Chen, "Parameter optimization of time-varying stiffness method for chatter suppression based on magnetorheological fluid-controlled boring bar," International Journal of Advanced Manufacturing Technology, vol. 46, no. 9-12, pp. 1071-1083, 2010.

[8] Q. H. Nguyen and S. B. Choi, "Optimal design of a novel hybrid MR brake for motorcycles considering axial and radial magnetic flux," Smart Materials and Structures, vol. 21, no. 5, Article ID 055003, 2012.

[9] Z. Ling, L. Xiaohui, and L. Yinong, "Optimization of magnetorheological damper based on a combination of objective function," Journal of Vibration Engineering (Chinese Journal), vol. 24, no. 6, pp. 600-605, 2011.

[10] Z. Parlak, T. Engin, and I. Çalli, "Optimal design of MR damper via finite element analyses of fluid dynamic and magnetic field," Mechatronics, vol. 22, no. 6, pp. 890-903, 2012.

[11] B. Yang, J. Luo, and L. Dong, "Magnetic circuit FEM analysis and optimum design for MR damper," International Journal of Applied Electromagnetics and Mechanics, vol. 33, no. 1-2, pp. 207-216, 2010.

[12] P. B. Nguyen and S. B. Choi, "A new approach to magnetic circuit analysis and its application to the optimal design of a bi-directional magnetorheological brake," Smart Materials and Structures, vol. 20, no. 12, Article ID 125003, 2011.

[13] T. Pranoto, K. Nagaya, Y. Ebara, and Q. Q. Long, "Vibration suppression device using permanent-electromagnet and MRF shear damper," Journal of Materials Processing Technology, vol. 181, no. 1-3, pp. 235-240, 2007.

[14] W. Hu, G. J. Hiemenz, G. Ngatu, and N. M. Wereley, "Performance evaluation of shear mode and flow mode magnetorheological (MR) energy absorbers for adaptive seat suspension systems," in Proceedings of the 53rd AIAA/ASME/ASCE/AHS/ASC Structures, Structural Dynamics and Materials Conference, Collection of Technical Papers, 2012. 

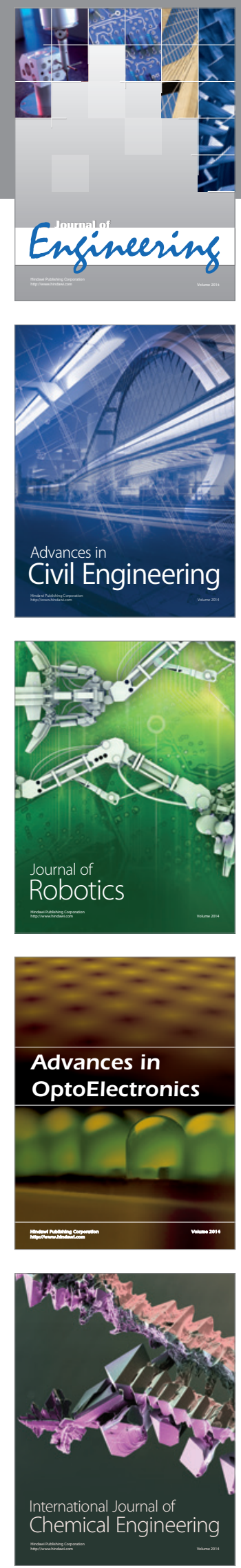

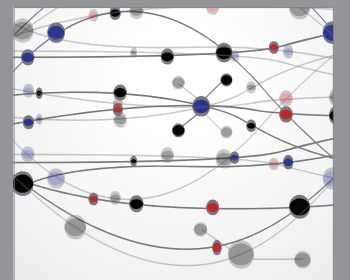

The Scientific World Journal
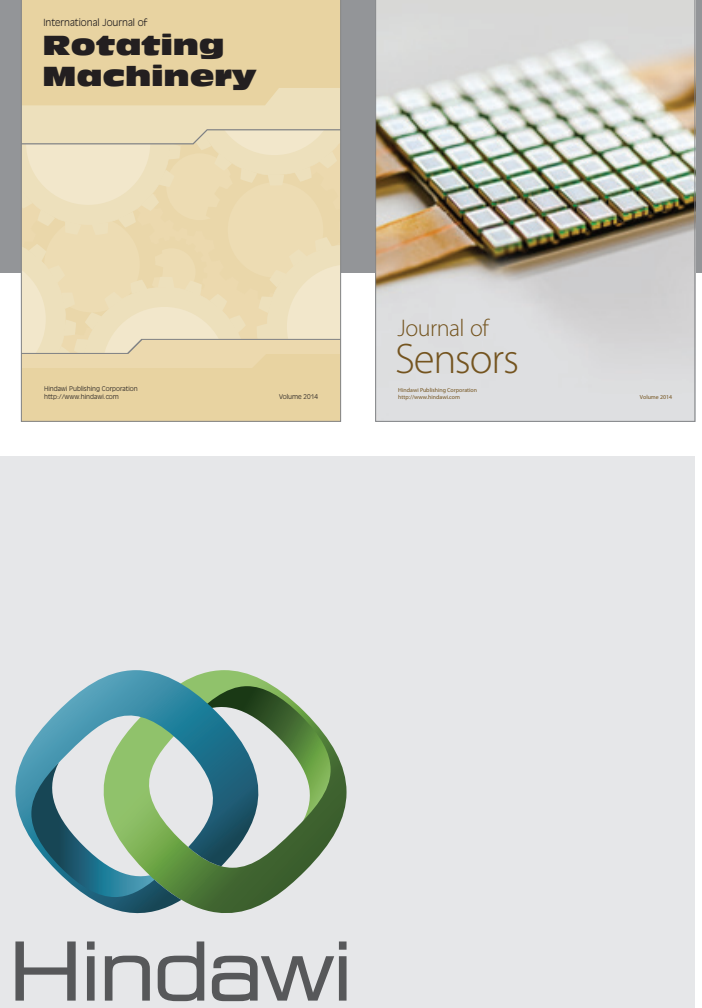

Submit your manuscripts at http://www.hindawi.com
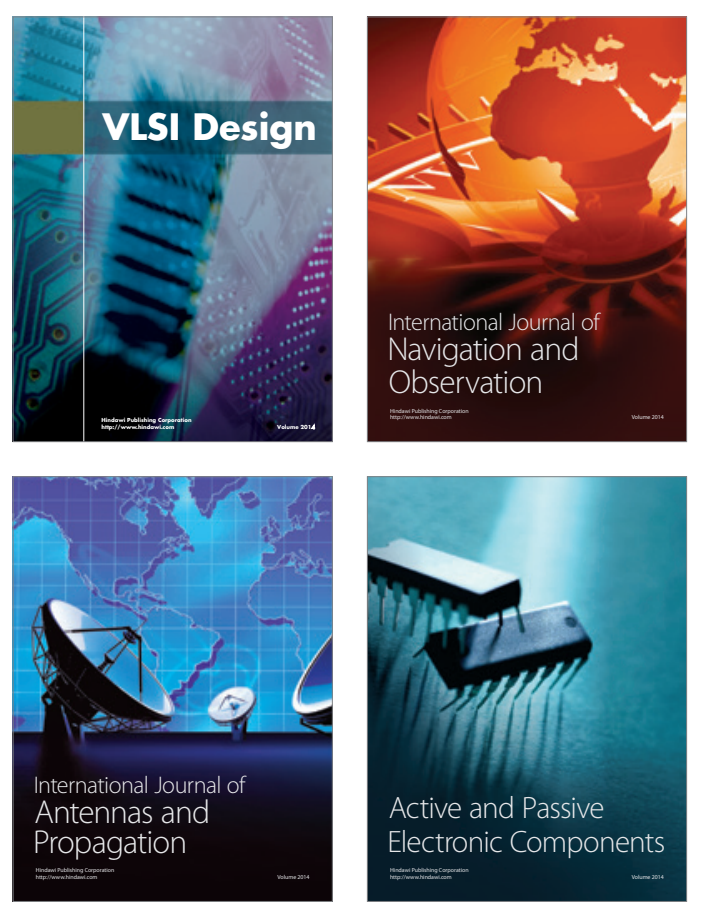
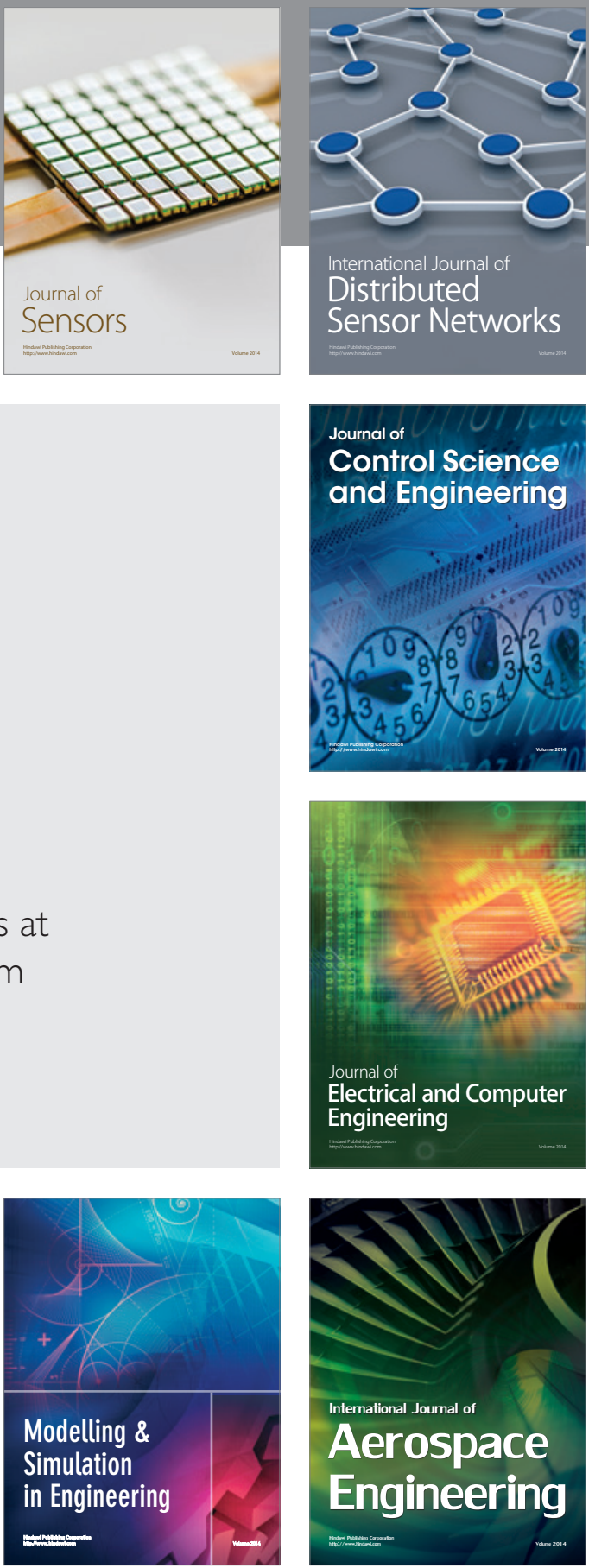

Journal of

Control Science

and Engineering
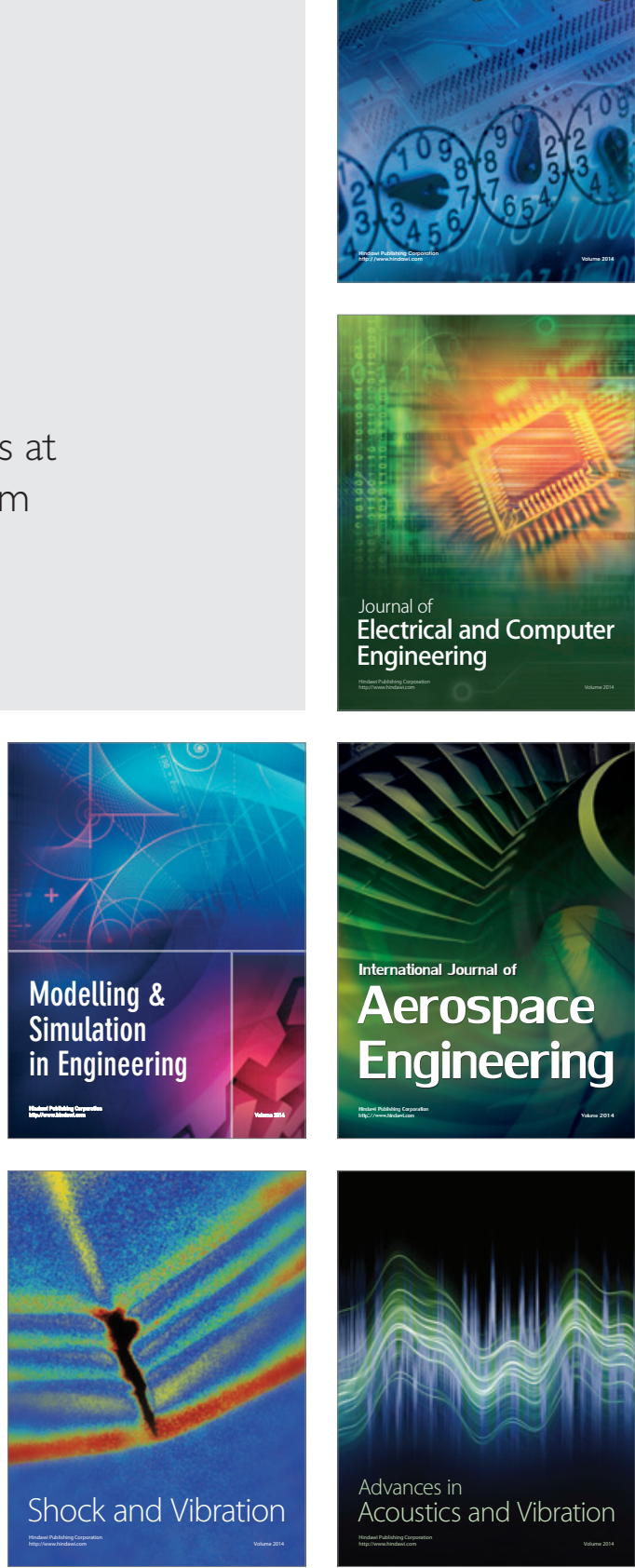